\title{
Strain engineering for high-level 5-aminolevulinic acid production in Escherichia coli
}

\author{
Dragan Miscevic ${ }^{1}$, Ju-Yi Mao ${ }^{2}$, Teshager Bitew Kefale ${ }^{1}$, Daryoush Abedi ${ }^{1}$, Murray Moo \\ young $^{1}$, and C. Perry Chou ${ }^{1}$ \\ ${ }^{1}$ University of Waterloo \\ ${ }^{2}$ National Taiwan Ocean University
}

May 5, 2020

\begin{abstract}
As issues surrounding depleting fossil fuels, climate change, and various other environmental impacts are becoming more prevalent, there is a growing interest in technological shifts toward a bio-based economy. Various advanced biotechnological tools have been developed to customize cell factories for the production of a wide range of complex fine chemicals from renewable feedstock. Herein, we report development of a microbial bioprocess for high-level and potentially economical production of 5aminolevulinic acid (5-ALA), a valuable non-proteinogenic amino acid with multiple applications in medical, agricultural, and food industries, using Escherichia coli as a cell factory. We first implemented the Shemin (i.e., C4) pathway for heterologous 5ALA biosynthesis in E. coli. To reduce, but not to abolish, the carbon flux toward essential tetrapyrrole/porphyrin biosynthesis, we applied Clustered Regularly Interspersed Short Palindromic Repeats interference (CRISPRi) to repress hemB expression, leading to extracellular 5-ALA accumulation. We then applied metabolic engineering strategies to direct more dissimilated carbon flux toward the key precursor of succinyl-CoA for enhanced 5-ALA biosynthesis. Using these engineered E. coli strains for bioreactor cultivation, we successfully demonstrated high-level 5-ALA biosynthesis solely from glycerol ( $~ 30 \mathrm{~g} \mathrm{l-1)}$ under both microaerobic and aerobic conditions, achieving up to $5.95 \mathrm{~g} \mathrm{l}-1$ (36.9\% yield) and $6.93 \mathrm{~g} \mathrm{l}-1$ (50.9\% yield) 5-ALA, respectively. This study represents one of the most effective bio-based production of 5-ALA from a structurally unrelated carbon to date, highlighting the importance of integrated strain engineering and bioprocessing strategies to enhance bio-based production.
\end{abstract}

\section{Introduction}

Increasing concerns over climate change and fossil fuel depletion have provoked a need for sustainable production of chemicals and fuels. One approach of particular attractiveness is the use of biological cell factories, such as microorganisms, as biocatalysts to drive chemical conversions from renewable and clean carbons, so called "bio-based production". The emergence of novel biotechnological tools for strain engineering, in particular systems biology, synthetic biology, metabolic engineering, and genetic/genomic engineering, has promoted the development of hyper strains for bio-based production (Choi et al. 2019). In addition to the sustainability, bio-based production can have economical potentials over synthetic organic chemistry particularly for the production of complex chemicals in the pharmaceutical and fine chemical industries, such as amino acids, organic acids, and vitamins (Keasling 2010).

5-Aminolevulinic acid (5-ALA) is a non-proteinogenic amino acid existing in most living organisms as a metabolic intermediate toward biosynthesis of essential tetrapyrrole/porphyrin pigment compounds, such as heme (Schlicke et al. 2015) (Fig. 1). In nature, there are two major metabolic routes for 5-ALA biosynthesis, i.e., (1) C4 (also known as Shemin) pathway (mainly existing in mammals, fungi, and purple sulphur bacteria), in which succinyl-CoA and glycine are structurally fused by 5 -aminolevulinate synthase (ALAS or HemA) to form 5-ALA (Kang et al. 2004), and (2) C5 pathway (existing in most bacteria, all archaea and 
plants), in which glutamate is converted to 5-ALA via three enzymatic reactions of ligation, reduction, and transamination catalyzed by glutamyl-tRNA synthase (GluTS), glutamyl-tRNA reductase (GluTR), and glutamate-1-semialdehyde-2,1-aminomutase (GSAM), respectively (Woodard and Dailey 1995).

Practically, 5-ALA has broad applications in many fields, such as medicine (Inoue 2017; Juzeniene et al. 2002), agriculture (Hotta et al. 1997), and food preservation (Li et al. 2016). Hence, technologies for 5-ALA production have been developed. While 5-ALA can be chemically derived from various precursors, such as levulinic acid (MacDonald 1974), tetrahydrofurfurylamine (Kawakami et al. 1991), 5-bromo esters (Ha et al. 1994), and $N$-furfurylphthalimide (Takeya et al. 1996), these synthetic approaches are deemed uneconomical and the production processes are often complicated for implementation with low yields (Kang et al. 2017). As a result, bio-based production of 5-ALA using either multi-enzyme systems (Meng et al. 2016) or various cell factories has been explored (Sasaki et al. 2002), in particular photosynthetic microorganisms, such asRhodobacter sphaeroides, Rhodopseudomonas palustris, and Chlorella sp. (Sasaki et al. 1995), Streptomyces coelicolor(Tran et al. 2019), Corynebacterium glutamicum (Zhang and Ye 2018), as well as genetically tractable Escherichia coli(Ding et al. 2017; Zhang et al. 2015; Zhang et al. 2019).

In this study, we explored strain engineering strategies for high-level 5-ALA production in E. coli. Native biosynthesis of 5-ALA inE. coli is achieved via the C5 pathway, which is adopted in most previous studies for 5-ALA biosynthesis using E. coli as a cell factory (Kang et al. 2011; Li et al. 2014; Zhang et al. 2015). However, this metabolic route is considered mechanistically complex and energetically ineffective, particularly upon high-level 5-ALA biosynthesis, as it requires multiple tightly regulated enzymes (Wang et al. 1999) and utilization of ATP/NADPH as limiting cofactors ( $\mathrm{Li}$ et al. 1989). On the other hand, for highlevel biosynthesis of a target metabolite, it is critical to ensure intracellular abundance of the corresponding precursors, i.e., succinyl-CoA/glycine for the Shemin pathway or glutamate for the C5 pathway in the 5ALA case. This is normally achieved by proper metabolic direction of the dissimilated carbon flux in key pathways, otherwise artificial supplementation of structurally related carbons, which are often expensive, becomes necessary. Considering the above technical aspects/limitations, we chose to implement the Shemin pathway into $E$. coli for heterologous 5-ALA biosynthesis with metabolic direction of the dissimilated carbon flux toward succinyl-CoA in the tricarboxylic acid (TCA) cycle. Moreover, without supplementation of structurally related carbons, glycerol was used as the sole carbon source for cultivation of engineered $E$. coli strains due to its low cost (Ciriminna et al. 2014) and highly reduced nature, generating approximately twice the number of reducing equivalents upon its degradation compared to traditional fermentable sugars (Murarka et al. 2008; Yazdani and Gonzalez 2007).

By initializing the formation of essential porphyrin compounds, 5-ALA is among the most conserved metabolites across all biological kingdoms (Petříčková et al. 2015; Yu et al. 2015). However, 5-ALA normally acts as a metabolic intermediate toward porphyrin biosynthesis with minimal accumulation, limiting its overproduction. Given the imperative physiological role of porphyrin compounds, attempting to accumulate 5-ALA by inactivation of the immediate post-5-ALA conversion catalyzed by 5-aminolevulinate dehydratase (HemB, encoded by hemB ) would abolish porphyrin biosynthesis (Fig. 1) and, therefore, be detrimental to the cells. Hence, instead of gene inactivation, we applied Clustered Regularly Interspersed Short Palindromic Repeats interference (CRISPRi) to repress hemB expression and, therefore, increase 5-ALA accumulation without imposing physiological impacts to the 5-ALA-producing cells. Using various engineered E. coli strains for bioreactor cultivation, we demonstrated high-level 5-ALA biosynthesis under both microaerobic and aerobic conditions with glycerol as a sole carbon source.

\section{Materials and Methods}

\section{Bacterial strains and plasmids}

Bacterial strains and deoxynucleic acids (DNAs) used in this study are listed Table 1. Genomic DNA from bacterial cells was isolated using the Blood \& Tissue DNA Isolation Kit (Qiagen, Hilden, Germany). Standard recombinant DNA technologies were applied for molecular cloning (Miller 1992). Taq DNA polymerase was obtained from New England Biolabs (Ipswich, MA, USA). All synthesized oligonucleotides were obtained 
from Integrated DNA Technologies (Coralville, IA, USA). DNA sequencing was conducted by the Centre for Applied Genomics at the Hospital for Sick Children (Toronto, Canada). E. coli BW25113 was the parental strain for derivation of all engineered strains in this study and E. coli DH5 $\alpha$ was used as a host for molecular cloning. Note that the $l d h A$ gene (encoding lactate dehydrogenase) was previously inactivated in BW25113, generating BW[?]ldhA (Srirangan et al. 2014).

For genetic implementation of the Shemin pathway in E. coli, thehemA gene was first amplified by polymerase chain reaction (PCR) using the primer set g-hemA and the genomic DNA of wild-typeRhodobacter sphaeroides DSM 158 as the template. The amplifiedhemA gene was Gibson-assembled with the PCRlinearized pK184 using the primer set g-pK-hemA to generate pK-hemA. The expression of the cloned hemA gene in the pK184 vector was under the control of the $\mathrm{P}_{l a c}$ promoter.

Knockouts of the genes, including $s d h A$ (encoding succinate dehydrogenase complex flavoprotein subunit A, SdhA) and $i c l R$ (encoding transcriptional AceBAK operon repressor, IclR), were introduced into BW[?] ldhA by P1 phage transduction (Miller 1992) using the appropriate Keio Collection strains (The Coli Genetic Stock Center, Yale University, New Haven, CT, USA) as donors (Baba et al. 2006). To eliminate the co-transduced FRT-Kn ${ }^{\mathrm{R}}$-FRT cassette, the transductants were transformed with pCP20 (Cherepanov and Wackernagel 1995), a temperature sensitive plasmid expressing a flippase (Flp) recombinase. Upon Flp-mediated excision of the $\mathrm{Kn}^{\mathrm{R}}$ cassette, a single Flp recognition site (FRT "scar site") was generated. Plasmid pCP20 was then cured by growing cells at $42^{\circ} \mathrm{C}$. The genotypes of derived knockout strains were confirmed by colony PCR using the appropriate verification primer sets listed in Table 1.

The expression of hemB was repressed by CRISPRi using various derived plasmids from pdcas9-bacteria (Addgene plasmid \# 44249) and pgRNA-bacteria (Addgene plasmid \# 44251). All synthesized oligonucleotide pairs have 60 nucleotides (nt), which includes 20 nthemB -targeting sequence, $20 \mathrm{nt}$ upstream and $20 \mathrm{nt}$ downstream sequences of pgRNA-bacteria vector (Fig. 2). They were ordered from Integrated DNA Technologies (Coralville, IA, USA) and annealed as described previously (Pengpumkiat et al. 2016), generating four double-stranded DNA fragments ofhemB -gRNA-L1, hemB -gRNA-L2, hemB -gRNA-L3, andhemB -gRNA-L4 (Table 1). All these four DNA fragments were individually Gibson-assembled with the PCRlinearized pgRNA-bacteria using the primer set g-pgRNA to generate pgRNA-L1, pgRNA-L2, pgRNA-L3, and pgRNA-L4, respectively (Table 1). The four hemB -repressed strains, i.e., DMH-L1, DMH-L2, DMH-L3, and DMH-L4, were developed by creating a triple-plasmid system (Fig. 2) containing pK-hemA, pdcas9bacteria, and the gRNA-containing plasmid (i.e., pgRNA-L1, pgRNA-L2, pgRNA-L3, or pgRNA-L4). For the control strain DMH-CT, the original pgRNA-bacteria plasmid without any hemB -targeting sequence was used as the third plasmid.

Media and bacterial cell cultivation

All medium components were obtained from Sigma-Aldrich Co. (St Louis, MO, USA) except yeast extract and tryptone which were obtained from BD Diagnostic Systems (Franklin Lakes, NJ, USA). E. coli strains, stored as glycerol stocks at $-80^{\circ} \mathrm{C}$, were streaked on lysogeny broth (LB; $10 \mathrm{~g} \mathrm{l}^{-1}$ tryptone, $5 \mathrm{~g} \mathrm{l}^{-1}$ yeast extract, and $5 \mathrm{~g} \mathrm{l}^{-1} \mathrm{NaCl}$ ) agar plates and incubated at $37^{\circ} \mathrm{C}$ for $14-16$ hours.

For shake-flask cultivations, single colonies were picked from LB plates to inoculate $30 \mathrm{ml} \mathrm{LB}$ medium in $125 \mathrm{ml}$ conical flasks. The cultures were shaken at $37^{\circ} \mathrm{C}$ and $280 \mathrm{rpm}$ in a rotary shaker (New Brunswick Scientific, NJ, USA) and used as seed cultures to inoculate $220 \mathrm{ml} \mathrm{LB}$ media at $1 \%$ (v/v) in $1 \mathrm{~L}$ conical flasks. This second seed culture was shaken at $37^{\circ} \mathrm{C}$ and $280 \mathrm{rpm}$ until a cell density of $0.80 \mathrm{OD}_{600}$ was reached. Cells were then harvested by centrifugation at $9,000 \times \mathrm{g}$ and $20^{\circ} \mathrm{C}$ for 10 minutes and resuspended in $30 \mathrm{ml}$ modified M9 production media. The suspended culture was transferred into a $125 \mathrm{ml}$ screwed cap plastic production flasks and incubated at $37^{\circ} \mathrm{C}$ at $280 \mathrm{rpm}$ in a rotary shaker. Unless otherwise specified, the modified M9 production medium contained $20 \mathrm{~g} \mathrm{l}^{-1}$ glycerol, $5 \mathrm{~g} \mathrm{l}^{-1}$ yeast extract, $10 \mathrm{mM} \mathrm{NaHCO}, 1 \mathrm{mM}$ $\mathrm{MgCl}_{2}, 5^{\text {th }}$ dilution of M9 salts mix (33.9 $\left.\mathrm{g} \mathrm{l}^{-1} \mathrm{Na}_{2} \mathrm{HPO}_{4}, 15 \mathrm{~g} \mathrm{l}^{-1} \mathrm{KH}_{2} \mathrm{PO}_{4}, 5 \mathrm{~g} \mathrm{l}^{-1} \mathrm{NH}_{4} \mathrm{Cl}, 2.5 \mathrm{~g} \mathrm{l}^{-1} \mathrm{NaCl}\right)$, $1,000^{\text {th }}$ dilution of Trace Metal Mix A5 $\left(2.86 \mathrm{~g} \mathrm{l}^{-1} \mathrm{H}_{3} \mathrm{BO}_{3}, 1.81 \mathrm{~g} \mathrm{l}^{-1} \mathrm{MnCl}_{2} * 4 \mathrm{H}_{2} \mathrm{O}, 0.222 \mathrm{~g} \mathrm{l}^{-1} \mathrm{ZnSO}_{4} * 7 \mathrm{H}_{2} \mathrm{O}\right.$, $\left.0.39 \mathrm{~g} \mathrm{l}^{-1} \mathrm{Na}_{2} \mathrm{MoO}_{4}{ }^{*} 2 \mathrm{H}_{2} \mathrm{O}, 79 \mu \mathrm{g} \mathrm{l}^{-1} \mathrm{CuSO}_{4}{ }^{*} 5 \mathrm{H}_{2} \mathrm{O}, 49.4 \mu \mathrm{g} \mathrm{l}{ }^{-1} \mathrm{Co}\left(\mathrm{NO}_{3}\right)_{2} * 6 \mathrm{H}_{2} \mathrm{O}\right)$, and was supplemented 
with $0.1 \mathrm{mM}$ isopropyl $\beta$-D-1-thiogalactopyranoside (IPTG). All shake-flask cultivation experiments were performed in triplicate.

For bioreactor cultivations, single colonies were picked from LB plates to inoculate $30 \mathrm{~mL}$ super broth (SB) medium (32 $\mathrm{g} \mathrm{l}^{-1}$ tryptone, $20 \mathrm{~g} \mathrm{l}^{-1}$ yeast extract, and $5 \mathrm{~g} \mathrm{l}^{-1} \mathrm{NaCl}$ ) in $125 \mathrm{~mL}$ conical flasks. The overnight cultures were shaken at $37^{\circ} \mathrm{C}$ and $280 \mathrm{rpm}$ in a rotary shaker (New Brunswick Scientific, NJ, USA) and used as seed cultures to inoculate $220 \mathrm{~mL} \mathrm{SB}$ media at $1 \%(\mathrm{v} / \mathrm{v})$ in $1 \mathrm{~L}$ conical flasks. This second seed culture was shaken at $37^{\circ} \mathrm{C}$ and $280 \mathrm{rpm}$ for $14-16$ hours. Cells were then harvested by centrifugation at $9,000 \times \mathrm{g}$ and $20^{\circ} \mathrm{C}$ for 10 minutes and resuspended in $50 \mathrm{~mL}$ fresh LB media. The suspended culture was used to inoculate a $1 \mathrm{~L}$ stirred tank bioreactor (containing two Rushton radial flow disks as impellers) (CelliGen 115, Eppendorf AG, Hamburg, Germany) at $37^{\circ} \mathrm{C}$ and $430 \mathrm{rpm}$. The semi-defined production medium in the batch bioreactor contained $30 \mathrm{~g} \mathrm{l}^{-1}$ glycerol, $0.23 \mathrm{~g} \mathrm{l}^{-1} \mathrm{~K}_{2} \mathrm{HPO}_{4}, 0.51 \mathrm{~g} \mathrm{l}^{-1} \mathrm{NH}_{4} \mathrm{Cl}, 49.8 \mathrm{mg} \mathrm{l}^{-1} \mathrm{MgCl}_{2}, 48.1$ $\mathrm{mg} \mathrm{l}^{-1} \mathrm{~K}_{2} \mathrm{SO}_{4}, 1.52 \mathrm{mg} \mathrm{l}^{-1} \mathrm{FeSO}_{4}, 0.055 \mathrm{mg} \mathrm{l}^{-1} \mathrm{CaCl}_{2}, 2.93 \mathrm{~g} \mathrm{l}^{-1} \mathrm{NaCl}, 0.72 \mathrm{~g} \mathrm{l}^{-1}$ tricine, $10 \mathrm{~g} \mathrm{l}^{-1}$ yeast extract, $10 \mathrm{mM} \mathrm{NaHCO}_{3}$, and 1,000th dilution (i.e., $\left.1 \mathrm{ml} \mathrm{l}^{-1}\right)$ trace elements $\left(2.86 \mathrm{~g} \mathrm{l}^{-1} \mathrm{H}_{3} \mathrm{BO}_{3}, 1.81 \mathrm{~g} \mathrm{l}^{-1} \mathrm{MnCl}_{2}\right.$ * $4 \mathrm{H}_{2} \mathrm{O}, 0.222 \mathrm{~g} \mathrm{l}^{-1} \mathrm{ZnSO}_{4} * 7 \mathrm{H}_{2} \mathrm{O}, 0.39 \mathrm{~g} \mathrm{l}^{-1} \mathrm{Na}_{2} \mathrm{MoO}_{4} * 2 \mathrm{H}_{2} \mathrm{O}, 79 \mu \mathrm{g} \mathrm{l} \mathrm{l}^{-1} \mathrm{CuSO}_{4} * 5 \mathrm{H}_{2} \mathrm{O}, 49.4 \mu \mathrm{g} \mathrm{l}{ }^{-1} \mathrm{Co}\left(\mathrm{NO}_{3}\right)_{2}$ * $6 \mathrm{H}_{2} \mathrm{O}$ ) (Neidhardt et al. 1974), and was supplemented with $0.1 \mathrm{mM}$ isopropyl $\beta$-D-1-thiogalactopyranoside (IPTG). Microaerobic and semiaerobic conditions were maintained by purging air into the headspace and bulk culture, respectively, at $0.1 \mathrm{vvm}$, designated as aeration level I (AL-I) and II (AL-II). Aerobic conditions were maintained by purging air into the bulk culture at $1 \mathrm{vvm}$ (AL-III). The $\mathrm{pH}$ of the production culture was maintained at $7.0 \pm 0.1$ with $30 \%(\mathrm{v} / \mathrm{v}) \mathrm{NH}_{4} \mathrm{OH}$ and $15 \%(\mathrm{v} / \mathrm{v}) \mathrm{H}_{3} \mathrm{PO}_{4}$.

Analysis

Culture samples were appropriately diluted with $0.15 \mathrm{M}$ saline solution for measuring cell density in $\mathrm{OD}_{600}$ using a spectrophotometer (DU520, Beckman Coulter, Fullerton, CA). Cell-free medium was prepared by centrifugation of the culture sample at $9,000 \times \mathrm{g}$ for 5 minutes, followed by filter sterilization using a 0.2 $\mu \mathrm{M}$ syringe filter. Extracellular metabolites and glycerol were quantified using high-performance liquid chromatography (HPLC) (LC-10AT, Shimadzu, Kyoto, Japan) with a refractive index detector (RID; RID10A, Shimadzu, Kyoto, Japan) and a chromatographic column (Aminex HPX-87H, Bio-Rad Laboratories, CA, USA). The HPLC column temperature was maintained at $35^{\circ} \mathrm{C}$ and the mobile phase was $5 \mathrm{mM}$ $\mathrm{H}_{2} \mathrm{SO}_{4}(\mathrm{pH} 2)$ running at $0.6 \mathrm{~mL} \mathrm{~min}{ }^{-1}$. The RID signal was acquired and processed by a data processing unit (Clarity Lite, DataApex, Prague, Czech Republic).

The 5-ALA titer in the cell-free medium was measured using a modified Ehrlich's reagent (Mauzerall and Granick 1956). The percentage yield of 5-ALA was defined as the mole (or mass) ratio of the produced 5-ALA to the theoretically maximal 5-ALA produced based on the consumed glycerol with a molar ratio of one-tothree. Note that one-mole succinyl-CoA (derived from two-mole glycerol) and one-mole glycine (derived from one-mole glycerol) are required to generate one-mole 5-ALA. The bulk level of secreted porphyrin compounds in the cell-free medium was estimated using a spectrophotometer at two specific wavelengths, i.e., $\mathrm{OD}_{405}$ (measuring Soret band) and $\mathrm{OD}_{495}$ (measuring Q-band).

Real-time quantitative reverse transcription PCR (qRT-PCR)

Cells used for RNA extraction were cultivated in $30 \mathrm{~mL}$ liquid $\mathrm{LB}$ medium at $37^{\circ} \mathrm{C}$ and harvested in the exponential growth phase. Total RNA of E. coli was extracted using the High Pure RNA Isolation Kit (Roche Diagnostics, Basel, Switzerland) according to manufacturer's instructions. Complementary DNAs (cDNAs) were synthesized using the High-Capacity cDNA Reverse Transcription Kit (ThermoFisher Scientific, MA). Sequence specific primers were used for reverse transcription ofhemB mRNA (i.e., q-hemB) and internal control $\operatorname{rrs} A$ (encoding ribosomal RNA 16S) mRNA (i.e., q-rrsA) (Table 1), at a final concentration of 1 $\mu \mathrm{M} .100 \mathrm{ng}$ of the total RNA was used in a $20 \mu \mathrm{L}$ reaction mixture. Real-time qRT-PCR was carried out using the Power SYBR B Green PCR Master Mix (ThermoFisher Scientific; MA) in an Applied Biosystems StepOnePlus System as per the manufacturer's instructions. All experiments were performed in duplicate.

\section{Results}




\section{Repression of hemB expression for extracellular 5-ALA accumulation}

We first implemented the Shemin pathway by episomal expression ofhemA from R. sphaeroides in BW[?]ldhA , deriving DMH. The effects of the implemented Shemin pathway were observed by comparing the two cultures of BW[?]ldhA and DMH in screw-cap shake-flasks containing $20 \mathrm{~g} \mathrm{l}^{-1}$ glycerol as the sole carbon source. While BW[?]ldhA generated no detectable levels of 5-ALA or porphyrin, DMH produced $0.44 \mathrm{~g} \mathrm{l}^{-1}$ 5 -ALA $(5.22 \%$ yield) with significant porphyrin biosynthesis (Fig. 3), suggesting that the Shemin pathway was active in DMH. In particular, the dark red color of the DMH culture (Fig. 3) suggests that most glycerol was converted to porphyrin pigments with limited 5-ALA accumulation.

We then aimed to reduce the carbon flux toward porphyrin biosynthesis in DMH by repressing the expression of hemB (Fig. 1), leading to a limited conversion of two 5-ALA molecules into porphobiliongen (PBG) and extracellular 5-ALA accumulation. We applied CRISPRi by designing fourhemB -targeting gRNAs with different relative expression efficiencies, generating four corresponding strains of DMH-L1, DMH-L2, DMHL3, and DMH-L4 (Fig. 3). Note that DMH-CT is the control strain without a hemB -specific gRNA for CRISPRi. While cell growth and glycerol consumption were somewhat affected by the presence of the tripleplasmid system, all hemB -repressed strains had significant extracellular 5-ALA accumulation compared to the control strain DMH-CT upon shake-flask cultivation. The minimal physiological impacts suggest that the hemB -repressed strains still had sufficient biosynthesis of essential porphyrins. In particular, DMHL1 and DMH-L4 had both titers and yields up to 4-5 fold higher than the control strain DMH-CT (Fig. 3). This significant increase in 5-ALA accumulation in DMH-L1 (1.26 $\left.\mathrm{g} \mathrm{l}^{-1}\right)$ and DMH-L4 (1.61 $\left.\mathrm{g} \mathrm{l}^{-1}\right)$ occurred simultaneous with considerably reduced levels of the relativehemB expression at $67 \%$ and $40 \%$, respectively (Fig. 2). Additional supporting evidence for the repressed hemB expression was reflected by the decrease in porphyrin biosynthesis in all fourhem $B$-repressed strains as the degrees of pigmentation and the corresponding absorbances (in 405 and $495 \mathrm{~nm}$ ) of the cell-free media were significantly lower than that of the control strain DMH-CT (Fig. 3). Given the lowest relative hemB expression and the most superior extracellular 5-ALA accumulation in DMH-L4 among allhemB -repressed strains, we used this strain for all subsequent experiments.

Effects of oxygenic condition on 5-ALA biosynthesis

5-ALA biosynthesis via the Shemin pathway requires succinyl-CoA as one of the two key precursors. Several metabolic pathways are involved in succinyl-CoA formation in E. coli, i.e., reductive TCA branch, oxidative TCA cycle, and glyoxylate shunt (Fig. 1). These metabolic pathways, along with cell growth and acetogenesis, can be sensitive to the oxygenic condition, which critically directs the dissimilated carbon flux toward succinyl-CoA for 5-ALA biosyntheses. To investigate such oxygenic effects, batch cultivation of the control strain DMH in a bioreactor was subject to three different levels of aeration, i.e., AL-I (microaerobic), AL-II (semiaerobic), and AL-III (aerobic) (Fig. 4). While cell growth and glycerol consumption were favored by oxygen exposure, 5-ALA biosynthesis was more effective under lower aeration levels, i.e., $0.53 \mathrm{~g} \mathrm{l}^{-1}(3.40 \%$ yield) under AL-I, $0.39 \mathrm{~g} \mathrm{l}^{-1}$ (2.44\% yield) under AL-II, and $0.31 \mathrm{~g} \mathrm{l}^{-1}$ (2.08\% yield) under AL-III, presumably because that more carbon was directed toward the succinyl-CoA node under microaerobic conditions. In addition, the reduced 5-ALA biosynthesis under aerobic conditions occurred with less porphyrin formation, reflected by a significantly less pigmentation of the culture medium. Note that, in addition to low levels of 5-ALA biosynthesis in DMH under all investigated culture conditions, acetate was the major side metabolite with high yields up to $75.9 \%$, implying a significant carbon spill at the acetyl-CoA node. The results suggest that 5-ALA biosynthesis in DMH was favored by lower oxygenic levels and potentially limited by acetogenesis.

Metabolic engineering to enhance 5-ALA biosynthesis under microaerobic conditions

To alleviate growth limitation and acetogenesis in DMH under AL-I, we derived a single-knockout mutant $\mathrm{DMH}[?] s d h A$, in which the oxidative TCA cycle was inactivated (Fig. 5-I). In addition to reduced acetogenesis, $\mathrm{DMH}[?] s d h A$ produced $0.94 \mathrm{~g} \mathrm{l}^{-1} 5$-ALA with $6.61 \%$ yield. With an increased carbon flux toward porphyrin biosynthesis, pigmentation of the $\mathrm{DMH}[?] s d h A$ culture medium was significantly enhanced. On 
the other hand, while glycerol consumption and cell growth were significantly retarded upon cultivation of the hemB -repressed strain DMH-L4 under AL-I, 5-ALA biosynthesis was drastically improved with much reduced acetogenesis, achieving $4.73 \mathrm{~g} \mathrm{l}^{-1} 5$-ALA with $32.0 \%$ yield (Fig. 5-II). The improved 5-ALA biosynthesis was also evidenced by significant reduction in pigmentation of the culture medium compared to DMH. Notably, compared to DMH[?]sdhA or DMH-L4, 5-ALA biosynthesis was further improved upon cultivation of DMH-L4[?] $s d h A$, in which the $s d h A$ mutation andhemB -repression was simultaneously introduced, under AL-I, achieving $5.95 \mathrm{~g} \mathrm{l}^{-1}$ 5-ALA with $36.9 \%$ yield (Fig. 5-III). These results suggest that the dissimilated carbon flux was directed toward the succinyl-CoA node for 5-ALA biosynthesis primarily via the reductive TCA branch under microaerobic conditions, and such carbon flux direction was rather effective upon simultaneous disruption of the oxidative TCA cycle and hemB repression. Finally, compared to DMH, higher levels of succinate, formate, and ethanol were observed upon cultivation of all engineered strains, i.e. DMH[?]sdhA , DMH-L4, and DMH-L4[?] sdhA , under microaerobic conditions for enhanced 5-ALA biosynthesis.

Metabolic engineering to enhance 5-ALA biosynthesis under aerobic conditions

We also explore 5-ALA biosynthesis under aerobic conditions, which often facilitate carbon utilization and cell growth. While the DMH-L4 culture under AL-III showed effective glycerol dissimilation and cell growth, biosynthesis of 5-ALA and porphyrins was much lower than that of the DMH-L4 culture under AL-I (Fig. 6-I vs. Fig. 5-II), suggesting a potential limitation in succinyl-CoA precursor under aerobic conditions. Nevertheless, the significant enhancing effects of hemB -repression on 5-ALA biosynthesis were still observable under aerobic conditions by comparing the two cultures of DMH-L4 and DMH under AL-III (Fig. 6-I vs. Fig. 4-III). To overcome the limitation in succinyl-CoA under AL-III, we derived another mutant of DMHL4[?]iclR with a deregulated glyoxylate shunt. Compared to the parental strain DMH-L4, DMH-L4[?]iclR had a much higher 5-ALA biosynthesis with effective glycerol dissimilation and cell growth under AL-III (Fig. 6-III vs. Fig 6-I), suggesting successful direction of the dissimilated carbon flux toward succinyl-CoA for 5ALA biosynthesis via the glyoxylate shunt under aerobic conditions. For more effective carbon flux direction, we derived another double mutant of DMH-L4[?] iclR [?] $s d h A$ with a disruptive oxidative TCA cycle such that the directed carbon flux at the succinate node via the glyoxylate shunt could be further directed toward succinyl-CoA via the reductive TCA branch. Compared to the parental strain DMH-L4[?]iclR, DMHL4[?]iclR [?] sdhA had even higher 5-ALA biosynthesis under AL-III (Fig. 6-V vs. Fig. 6-III), achieving $6.93 \mathrm{~g} \mathrm{l}^{-1}$ 5-ALA with $50.9 \%$ yield, though the single mutation of [?] $\operatorname{sdh} A$ appeared to be rather harmful to cell physiology and, therefore, culture performance (Fig. 6-II vs. Fig. 6-I). Note that the 5-ALA yield for DMH-L4[?] sdhA [?]iclR was 3.4-fold that for DMH-L4 and 1.3-fold that for DMH-L4[?]iclR . Also, note that the significant enhancing effects of hemB -repression on 5-ALA biosynthesis were further confirmed by comparing the two cultures of DMH-L4[?]iclR [?] sdhA and DMH[?]iclR [?]sdhA under AL-III (Fig. 6-V vs. Fig. 6-IV). On the other hand, the successful carbon flux direction toward the Shemin pathway via the glyoxylate shunt and reductive TCA branch for biosynthesis of both 5-ALA and porphyrin pigments can be also observed by comparing the two cultures of DMH[?]iclR [?]sdhA and DMH under AL-III (Fig. 6 -IV vs. Fig. 4-III). These results successfully demonstrated the consolidated strategy based on carbon flux redirection in the TCA cycle toward the Shemin pathway with repressed hemB expression to enhance 5-ALA biosynthesis.

\section{Discussion}

While E. coli has the native C5 pathway for 5-ALA biosynthesis, we herein adopted the exogenous Shemin pathway since the intracellular level of the key precursor succinyl-CoA can be metabolically manipulated. Being a metabolic intermediate in the pathway for biosynthesis of essential porphyrins, 5-ALA hardly accumulates inE. coli . To prevent intracellular drainage of 5-ALA, we explored gene knockout of hemB , but failed to derive the corresponding mutant strain (data now shown), confirming that hemB is an essential gene. Hence, CRISPRi was applied to repress hemBexpression, particularly in DMH-L1 and DMH-L4, such that 5-ALA could accumulate as a result of its reduced conversion. Our results further suggest that 5-ALA acts as a committed precursor for porphyrin biosynthesis. Despite the increased extracellular 5-ALA accumulation associated with the repressed hemB expression, suppression of carbon flux toward essential 
porphyrin biosynthesis somewhat impacted cell growth upon cultivation.

While the effects of oxygen supply on 5-ALA biosynthesis were investigated in several organisms (Nishikawa et al. 1999; Yu et al. 2015), little effort on this front was made for E. coli. With the implemented the Shemin pathway, 5-ALA biosynthesis in E. coli can critically depend on the availability of the key precursor of succinyl-CoA, whose formation is rather oxygen-sensitive. In E. coli, succinate (and, therefore, succinyl-CoA) can be derived via three oxygen-dependent pathways in the TCA cycle, i.e., (i) reductive TCA branch, (ii) oxidative TCA cycle, and (iii) glyoxylate shunt (Fig. 1) (Cheng et al. 2013). Under anaerobic conditions, succinate accumulates as an end-product of mixed acid fermentation via the reductive TCA branch (Thakker et al. 2012). Although the reductive TCA branch can potentially yield high-level succinate, this pathway is generally unfavorable due to the limited availability of reducing equivalents (Skorokhodova et al. 2015). Under aerobic conditions, succinate is normally used up as a metabolic intermediate of the oxidative TCA cycle without accumulation, except for the conditions of oxidative stress and/or acetate/fatty-acid consumption under which succinate can be aerobically derived via the operational glyoxylate shunt (Thakker et al. 2012). Using the control strain DMH, the effects of oxygenic conditions on 5-ALA biosynthesis were systematically investigated for cell cultivation under microaerobic (AL-I), semiaerobic (AL-II), and aerobic (AL-III) conditions. Note that glycerol utilization and cell growth were severely retarded when DMH was cultivated under a strict anaerobic condition (data not shown). Our results show that biosynthesis of 5-ALA and porphyrins was favored by microaerobic conditions, though the low oxygenic level could trigger high-level acetogenesis and the associated physiological impacts. The results suggest that, in $\mathrm{DMH}$, most of the dissimilated carbon flux was channeled into the Shemin pathway for biosynthesis of 5-ALA and porphyrins via the reductive TCA branch; and the oxidative TCA cycle and glyoxylate shunt contributed minimally toward such carbon flux channeling. Compared to $\mathrm{DMH}$, blocking the oxidative TCA cycle in $\mathrm{DMH}[?]$ sdhAcould potentially channel more dissimilated carbon flux toward succinyl-CoA via the reductive TCA branch under AL-I and, therefore, improve biosynthesis of 5-ALA and porphyrins with much reduced acetogenesis. With more dissimilated carbon flux channeling into the Shemin pathway, porphyrin biosynthesis was further reduced by repressing hemB expression to enhance 5-ALA accumulation in DMH-L4[?] $\operatorname{sdh} A$ under AL-I, achieving $5.95 \mathrm{~g} \mathrm{l}^{-1} 5$-ALA and with $36.9 \%$ yield while minimizing porphyrin biosynthesis. Note that inactivating the oxidative TCA cycle and/or repressinghemB expression resulted in uncommon accumulation of formate with reduced acetogenesis, compared to the control strain DMH. Such observation suggests that, under these genetic backgrounds and microaerobic conditions, pyruvate formate lyase (PFL; via which formate is coproduced) could be more active than pyruvate dehydrogenase (PDH) for the conversion of pyruvate to acetyl-CoA. In E. coli, PDH and PFL are responsible for decarboxylation of pyruvate to form acetyl-CoA under aerobic and anaerobic conditions, respectively (Wang et al. 2010). It was also reported that acetate and formate could induce opposite proteome responses in $E$. coli as most proteins induced by one of these two acids are repressed by the other (Kirkpatrick et al. 2001). While the control strain DMH had a low-level biosynthesis of 5-ALA and porphyrins under aerobic conditions such as AL-III, implying a limited carbon flux contribution from the oxidative TCA cycle (and, therefore, limited succinyl-CoA precursor) into the Shemin pathway, repressing hemB expression could also increase 5-ALA accumulation significantly in DMH-L4. Inactivating the TCA oxidative cycle in DMHL4[?]sdhA significantly retarded cell growth with limited glycerol dissimilation, cell growth, and metabolite production under AL-III, suggesting the critical metabolic roles of the TCA oxidative cycle for biomass formation and biosynthesis under aerobic conditions as per previous observations (Guest 1981; Steinsiek et al. 2011). To resolve the apparent succinyl-CoA limitation under AL-III, we explored channeling of the dissimilated carbon flux via a deregulated glyoxylate shunt by mutating iclR in DMH-L4[?]iclR and observed a significantly enhanced 5-ALA biosynthesis. Nevertheless, with an active TCA oxidative cycle in DMH-L4[?] iclR, the carbon flux arising from the deregulated glyoxylate shunt could divert at the succinate node to either the oxidative or re- 
ductive TCA direction. Importantly, the flux diversion could be prevented with the dissimilated carbon being effectively directed into the Shemin pathway by further mutating $s d h A$ for inactivation of the oxidative TCA cycle in DMH-L4[?] sdhA[?]iclR, achieving $6.93 \mathrm{~g} \mathrm{l}^{-1} 5$-ALA with $50.9 \%$ yield upon its cultivation under AL-III while minimizing porphyrin biosynthesis. Such carbon flux rerouting effects under AL-III could also be observed by the enhanced biosynthesis of 5-ALA and porphyrins (reflected by significant pigmentation of the culture medium in Fig. 6-IV) in $\mathrm{DMH}[?] \operatorname{sdh} A[?] i c l R$ compared to DMH. Additionally, note that the retarded glycerol utilization and cell growth for DMH-L $4 \Delta s d h A$ could be complemented by the iclRmutation, suggesting that both the oxidative TCA cycle and glyoxylate shunt contribute to active TCA operation for sustained cell growth and metabolic biosynthesis under aerobic conditions. While this study successfully demonstrated high-level biosynthesis of 5-ALA from structurally unrelated carbons under both microaerobic and aerobic conditions, the overall culture performance was limited by significant acetogenesis, particularly during extended fedbatch cultivation (data not shown).

\section{Funding information}

This work was supported by the following Government of Canada grants: (1 ) Natural Sciences and Engineering Research Council (NSERC) Strategic Partnership grant 430106-12; and (2 ) Canada Research Chair (CRC) grant 950-211471

\section{Competing financial interests}

The authors declare no competing financial interests.

\section{References}

Akawi, L., Srirangan, K., Liu, X., Moo-Young, M., \& Chou, C. P. (2015). Engineering Escherichia coli for high-level production of propionate. Journal of Industrial Microbiology \& Biotechnology , 42, 1057-1072.

Baba, T., Ara,T., Hasegawa, M., Takai, Y., Okumura, Y., Baba, M., Datsenko, K. A., Tomita, M., Wanner, B. L., \& Mori, H. (2006). Construction of Escherichia coli K-12 in-frame, single-gene knockout mutants: the Keio collection.Molecular Systems Biology , 2, 1-11.

Cheng, K-K., Wang, G-Y., Zeng, J., \& Zhang, J-A. (2013). Improved Succinate Production by Metabolic Engineering. BioMed Research International , 2013, 1-12.

Cherepanov, P. P., \& Wackernagel, W. (1995). Gene disruption in Escherichia coli : TcR and KmR cassettes with the option of Flp-catalyzed excision of the antibiotic-resistance determinant. Gene , 158, 9-14.

Choi, K. R., Jang, W. D., Yang, D., Cho, J. S., Park, D., \& Lee, S. Y. (2019). Systems Metabolic Engineering Strategies: Integrating Systems and Synthetic Biology with Metabolic Engineering. Trends in Biotechnology , 37, 817-837.

Ciriminna, R., Pina, C. D., Rossi, M., \& Pagliaro, M. (2014). Understanding the glycerol market.European Journal of Lipid Science \& Technology, 116, 1432-1439.

Datsenko, K. A., \& Wanner, B. L. (2000). One-step inactivation of chromosomal genes in Escherichia coli K-12 using PCR products. Proceedings of the National Academy of Sciences , 97, 6640-6645.

Ding, W., Weng, H., Du, G., Chen, J., \& Kang, Z. (2017). 5-Aminolevulinic acid production from inexpensive glucose by engineering the C4 pathway in Escherichia coli. Journal of Industrial Microbiology $\mathcal{B}^{3}$ Biotechnology , 44, 1127-1135.

Guest, J. R. (1981). Partial replacement of succinate dehydrogenase function by phage- and plasmid-specified fumarate reductase in Escherichia coli .Journal of General Microbiology, 122, 171-179.

Ha, H-J., Lee, S-K., Ha, Y-J., \& Park, J-W. (1994). Selective Bromination of Ketones. A Convenient Synthesis of 5-Aminolevulinic Acid. Synthetic Communications , 24, 2557-2562. 
Hotta, Y., Tanaka, T., Takaoka, H., Takeuchi, Y., Konnai, M. (1997). Promotive effects of 5-aminolevulinic acid on the yield of several crops. Plant Growth Regulation, 22, 109-114.

Inoue K. (2017). 5-Aminolevulinic acid-mediated photodynamic therapy for bladder cancer.International Journal of Urology , 24, 97-101.

Jobling, M. G., \& Holmes, R. K. (1990). Construction of vectors with the p15a replicon, kanamycin resistance, inducible lac $Z$ alpha and pUC18 or pUC19 multiple cloning sites. Nucleic Acids Research , 18, 5315-5316.

Juzeniene, A., Juzenas, P., Iani, V., \& Moan, J. (2002). Topical Application of 5-Aminolevulinic Acid and its Methylester, Hexylester and Octylester Derivatives: Considerations for Dosimetry in Mouse Skin Model. Photochemistry 83 Photobiology , 76, 329-334.

Kang, D-K., Kim, S., Chi, W. J., Hong, S. K., Kim, H. K., \& Kim, H. U. (2004). Cloning and expression of the Rhodobacter capsulatus hemA gene in E. coli for the production of 5-aminolevulinic acid. Journal of Microbiology \& Biotechnology, 14, 1327-1332.

Kang, Z., Ding, W., Gong, X., Liu, Q., Du, G., \& Chen, J. (2017). Recent advances in production of 5aminolevulinic acid using biological strategies. World Journal of Microbiology \&3 Biotechnology , 33, 200-207.

Kang, Z., Wang, Y., Gu, P., Wang, Q., \& Qi, Q. (2011). Engineering Escherichia coli for efficient production of 5-aminolevulinic acid from glucose. Metabolic Engineering , 13, 492-498.

Kawakami, H., Ebata, T., \& Matsushita, H. (1991). A New Synthesis of 5-Aminolevulinic Acid.Agricultural \& Biological Chemistry, 55, 1687-1688.

Keasling J. (2010). Manufacturing Molecules Through Metabolic Engineering. Science (New York, N.Y.), $330,1355-1358$.

Kirkpatrick, C., Maurer, L. M., Oyelakin, N. E., Yoncheva, Y. N., Maurer, R., \& Slonczewski, J. L. (2001). Acetate and formate stress: opposite responses in the proteome of Escherichia coli . Journal of Bacteriology , 183, 6466-6477.

Li, F., Wang, Y., Gong, K., Wang, Q., Liang, Q., \& Qi, Q. (2014). Constitutive expression of RyhB regulates the heme biosynthesis pathway and increases the 5-aminolevulinic acid accumulation in Escherichia coli . FEMS Microbiology Letters, 350, 209-215.

Li, J. M., Brathwaite., O., Cosloy., S. D., \& Russell., C. S. (1989). 5-Aminolevulinic acid synthesis inEscherichia coli . Journal of Bacteriology, 171, 2547-2552.

Li, Y., Li, Z., \& Wang, L. (2016). Applications of 5-aminolevulinic acid on the physiological and biochemical characteristics of strawberry fruit during postharvest cold storage. Ciência Rural , 46, 2103-2109.

MacDonald, S. (1974). Methyl 5-bromolevulinate. Canadian Journal of Chemistry, 52, 3257-3258.

Mauzerall, D., \& Granick, S. (1956). The occurrence and determination of $\delta$-aminolevulinic acid and porphobilinogen in urine. Journal of Biological Chemistry, 219, 435-446.

Meng, Q., Zhang, Y., Ju, X., Ma, C., Ma, H., Chen, J., Zheng, P., Sun, J., Zhu, J., Ma, Y., Zhao, X., \& Chen, T. (2016). Production of 5-aminolevulinic acid by cell free multi-enzyme catalysis. Journal of Biotechnology , 226, 8-13.

Miller, J. H. (1992). A short course in bacterial genetics: a laboratory manual and handbook forEscherichia coli and related bacteria. NY: Cold Spring Harbor Laboratory Press.

Murarka, A., Dharmadi, Y., Yazdani, S. S., Gonzalez, R. (2008). Fermentative Utilization of Glycerol by Escherichia coli and Its Implications for the Production of Fuels and Chemicals. Applied \&3 Environmental Microbiology , 74, 1124-1135. 
Neidhardt, F. C., Bloch, P. L., Smith, D. F. (1974). Culture Medium for Enterobacteria. Journal of Bacteriology , 119, 736-747.

Nishikawa, S., Watanabe, K., Tanaka, T., Miyachi, N., Hotta, Y., \& Murooka, Y. (1999). Rhodobacter sphaeroides mutants which accumulate 5-aminolevulinic acid under aerobic and dark conditions. Journal of Bioscience \& Bioengineering , 87, 798-804.

Pengpumkiat, S., Koesdjojo, M., Rowley, E. R., Mockler, T. C., \& Remcho, V. T. (2016). Rapid Synthesis of a Long Double-Stranded Oligonucleotide from a Single-Stranded Nucleotide Using Magnetic Beads and an Oligo Library. PloS One, 11, 1-10.

Petrríčková, K., Chroňáková, A., Zelenka, T., Chrudimský, T., Pospíšil, S. Petříček, M., \& Krištůfek, V. (2015). Evolution of cyclizing 5-aminolevulinate synthases in the biosynthesis of actinomycete secondary metabolites: outcomes for genetic screening techniques. Frontiers in Microbiology , 6, 1-15.

Qi, L. S., Larson, M. H., Gilbert, L. A., Doudna, J. A., Weissman, J. S., Arkin, A. P., Lim, W. A. (2013). Repurposing CRISPR as an RNA-guided platform for sequence-specific control of gene expression. Cell, $152,1173-1183$.

Sasaki, K., Watanabe, K., Tanaka, T., Hotta, Y., \& Nagai, S. (1995). 5-Aminolevulinic acid production by Chlorella sp. during heterotrophic cultivation in the dark. World Journal of Microbiology $\&$ Biotechnology , 11, 361-362.

Sasaki, K., Watanabe, M., Tanaka, T., \& Tanaka, T. (2002). Biosynthesis, biotechnological production and applications of 5-aminolevulinic acid. Applied Microbiology \&3 Biotechnology , 58, 23-29.

Schlicke, H., Richter, A., Rothbart, M., Brzezowski, P., Hedtke, B., \& Grimm, B. (2015). Function of Tetrapyrroles, Regulation of Tetrapyrrole Metabolism and Methods for Analyses of Tetrapyrroles. Procedia Chemistry , 14, 171-175.

Skorokhodova, A. Y., Morzhakova, A. A., Gulevich, A. Y., \& Debabov, V. G. (2015). Manipulating pyruvate to acetyl-CoA conversion in Escherichia coli for anaerobic succinate biosynthesis from glucose with the yield close to the stoichiometric maximum. Journal of Biotechnology, 214, 33-42.

Srirangan, K., Liu, X., Westbrook, A., Akawi, L., Pyne, M. E., Moo-Young, M., \& Chou, C. P. (2014). Biochemical, genetic, and metabolic engineering strategies to enhance coproduction of 1-propanol and ethanol in engineered Escherichia coli . Applied Microbiology \& Biotechnology , 98, 9499-9515.

Steinsiek, S., Frixel, S., Stagge, S., \& Bettenbrock, K. (2011). Characterization of E. coli MG1655 and frdA and $s d h C$ mutants at various aerobiosis levels. Journal of Biotechnology , 154, 35-45.

Takeya, H., Ueki, H., Miyanari, S., Shimizu, T., \& Kojima, M. (1996). A new synthesis of 5-aminolevulinic acid via dye-sensitized oxygenation of N-furfurylphthalimide. Journal of Photochemistry and Photobiology A: Chemistry, 94, 167-171.

Thakker, C., Martinez, I., San, K. Y., \& Bennett, G. N. (2012). Succinate production in Escherichia coli . Biotechnology Journal , 7, 213-224.

Tran, N. T., Pham, D. N., \& Kim, C-J. (2019). Production of 5-aminolevulinic Acid by RecombinantStreptomyces coelicolor Expressing hemA fromRhodobacter sphaeroides . Biotechnology 85 Bioprocess Engineering , 24, 488-499.

Wang, L., Elliott, M., \& Elliott, T. (1999). Conditional Stability of the HemA Protein (Glutamyl-tRNA Reductase) Regulates Heme Biosynthesis in Salmonella typhimurium .Journal of Bacteriology , 181, 12111219.

Wang, Q., Ou, M. S., Kim, Y., Ingram, L. O., \& Shanmugam, K. T. (2010). Metabolic flux control at the pyruvate node in an anaerobic Escherichia coli strain with an active pyruvate dehydrogenase. Applied $\mathscr{E}$ Environmental Microbiology, 76, 2107-2114. 
Woodard, S. I., \& Dailey, H. A. (1995). Regulation of Heme Biosynthesis in Escherichia coli .Archives of Biochemistry \& Biophysics, 316, 110-115.

Yazdani, S. S., \& Gonzalez, R. (2007). Anaerobic fermentation of glycerol: a path to economic viability for the biofuels industry. Current Opinion in Biotechnology, 18, 213-219.

Yu, X., Jin, H., Liu, W., Wang, Q., \& Qi, Q. (2015). Engineering Corynebacterium glutamicum to produce 5-aminolevulinic acid from glucose. Microbial Cell Factories, 14, 1-10.

Zhang, B., \& Ye, B-C. (2018). Pathway engineering in Corynebacterium glutamicum S9114 for 5aminolevulinic acid production. 3 Biotech , 8, 247.

Zhang, J., Kang, Z., Chen, J., \& Du, G. (2015). Optimization of the heme biosynthesis pathway for the production of 5-aminolevulinic acid in Escherichia coli .Scientific Reports , 5, 1-7.

Zhang, J., Weng, H., Zhou, Z., Du, G., \& Kang, Z. (2019). Engineering of multiple modular pathways for high-yield production of 5-aminolevulinic acid in Escherichia coli . Bioresource Technology , 274, 353-360.

Table captions

E. coli strains, plasmids, and oligonucleotides used in this study

\section{Figure captions}

1. Schematic representation of the natural metabolism and the implemented Shemin pathway for 5-ALA and porphyrin biosynthesis inE. coli from glycerol. Metabolic pathways outlined: glycolysis, glycine biosynthesis, pyruvate carboxylation, and oxidative TCA cycle (in black); glyoxylate shunt in the TCA cycle (in light brown); reductive branch of TCA cycle (in blue); Shemin pathway (in green); porphyrin biosynthesis (in red). Colored proteins: mutations (in red); overexpression (in purple). Metabolite abbreviations: 5,10-MTH, 5,10-methenyltetrahydrofolic acid; 5-ALA, 5aminolevulinic acid; 3-PG, 3-phosphoglycerate; 3-PP, 3-phosphooxypyruvate; O-P-Serine, O-phosphoL-serine; PBG, porphobilinogen; PEP, phosphoenolpyruvate. The number of carbon atoms for each metabolite is specified in orange. Protein abbreviations: AceA, isocitrate lyase; AceB, malate synthase A; AceK, isocitrate dehydrogenase kinase/phosphatase; AckA, acetate kinase; AdhE, aldehydealcohol dehydrogenase; FHL, formate hydrogenlyase; HemA, 5-aminolevulinate synthase; HemB, 5aminolevulinate dehydratase; IclR, AceBAK operon repressor; IDH, isocitrate dehydrogenase; IDH-P, isocitrate dehydrogenase-phosphate; LdhA, lactate dehydrogenase A; PC, pyruvate carboxylase; PckA, phosphoenolpyruvate carboxykinase; PDH, pyruvate dehydrogenase; PFL, pyruvate formate-lyase; PK, pyruvate kinase; PPC, phosphoenolpyruvate carboxylase; Pta, phosphotransacetylase; SdhA, succinate dehydrogenase complex (subunit A).

2. Design strategy for CRISPRi-mediated hemB repression .The three plasmids with their major genetic features, such as promoters, selection markers, key genes, are shown. The design ofhemB -targeting sequences and their associated interacting spots in the hemB gene (i.e., L1, L2, L3, and L4) and the predicted repression efficiencies (numbers in parenthesis) are shown. The resulting hemB -repressed strains, i.e., DMH-CT (control), DMH-L1, DMH-L2, DMH-L3, and DMH-L4, were characterized for quantification of the relative hemB expression using qRT-PCR. All qRT-PCR values are reported as means $\pm \mathrm{SD}(\mathrm{n}=2)$.

3. Shake-flask cultivation of hemB-repressed strains for 5-ALA accumulation. Strains compared include BW[?]ldhA, DMH, DMH-CT, DMH-L1, DMH-L2, DMH-L3, and DMH-L4. Results of the $48 \mathrm{~h}$ shake-flask cultivation in (a ) cell density $\left(\mathrm{OD}_{600}\right)$, (b ) glycerol consumption, (c ) 5-ALA titer and percentage yield, and (d ) porphyrin biosynthesis (represented by the absorbance readings of the Soret peak $\left(\mathrm{OD}_{405}\right)$ and $\mathrm{Q}$-band $\left(\mathrm{OD}_{495}\right)$ and the images of cell-free media) are shown. All values are reported as means $+-\mathrm{SD}(\mathrm{n}=3)$.

4. Bioreactor cultivation of DMH for 5-ALA biosynthesis under different oxygenic conditions. Time profiles of cell density $\left(\mathrm{OD}_{600}\right)$, glycerol consumption and metabolite production profiles, 
acetate and 5-APA percentage yields, and porphyrin biosynthesis (represented by the absorbance readings of the Soret peak in $\mathrm{OD}_{405}$ and Q-band in $\mathrm{OD}_{495}$ and the images of cell-free media) are shown. The percentage yields of acetate and 5-ALA are calculated based on the consumed glycerol at the end of cultivation. (I ) AL-I: microaerobic, (II ) AL-II: semi-aerobic, and (III ) AL-III: aerobic. All values are reported as means $+-\mathrm{SD}(\mathrm{n}=2)$.

5. Bioreactor cultivation of engineered $E$. coli for 5-ALA biosynthesis under microaerobic (AL-I) conditions. Strains compared include DMH[?]sdhA, DMH-L4, and DMH-L4[?]sdhA . Time profiles of cell density $\left(\mathrm{OD}_{600}\right)$, glycerol consumption and metabolite production profiles, acetate and 5-APA percentage yields, and porphyrin biosynthesis (represented by the absorbance readings of the Soret peak in $\mathrm{OD}_{405}$ and Q-band in $\mathrm{OD}_{495}$ and the images of cell-free media) are shown. The percentage yields of acetate and 5-ALA are calculated based on the consumed glycerol at the end of cultivation. (I ) $\mathrm{DMH}[?] s d h A,(\mathbf{I I})$ DMH-L4, and (III ) DMH-L4[?]sdhA. All values are reported as means +$\mathrm{SD}(\mathrm{n}=2)$.

6. Bioreactor cultivation of engineered $E$. coli for 5-ALA biosynthesis under aerobic (ALIII) conditions. Strains compared include DMH-L4, DMH-L4[?] sdhA , DMH-L4[?]iclR, DMH[?] sdhA [?]iclR, and DMH-L4[?] sdhA [?]iclR . Time profiles of cell density $\left(\mathrm{OD}_{600}\right)$, glycerol consumption and metabolite production profiles, acetate and 5-APA percentage yields, and porphyrin biosynthesis (represented by the absorbance readings of the Soret peak in $\mathrm{OD}_{405}$ and Q-band in $\mathrm{OD}_{495}$ and the images of cell-free media) are shown. The percentage yields of acetate and 5-ALA are calculated based on the consumed glycerol at the end of cultivation. (I ) DMH-L4 (II ) DMH-L4[?]sdhA , (III ) DMHL4[?]iclR, (IV ) DMH[?]sdhA [?]iclR, and (V ) DMH-L4[?] sdhA [?]iclR . All values are reported as means $+-\mathrm{SD}(\mathrm{n}=2)$.

\begin{tabular}{|c|c|c|}
\hline Name & $\begin{array}{l}\text { Description, relevant } \\
\text { genotype or primer/oligo } \\
\text { sequence }\left(5^{\prime} 3^{\prime}\right)\end{array}$ & Source \\
\hline \multicolumn{3}{|l|}{ E. coli host strains } \\
\hline DH $5 \alpha$ & $\begin{array}{l}\text { F-, } \epsilon \nu \delta A 1, \gamma \lambda \nu^{\prime \prime 44, ~ \tau \eta-1, ~} \rho \in \varsigma A 1, \\
\rho \in \lambda A 1, \nu \psi \rho A 96, \delta \epsilon o P, \nu \cup \pi \Gamma \\
\varphi 80 \delta \lambda a \varsigma Z[;] a \varsigma Z \delta \lambda a \delta \lambda a \varsigma Z \Psi A- \\
a \rho \gamma \Phi) \Upsilon 169, \eta \sigma \delta P 17(\rho K-\mu K \\
+), \lambda-\end{array}$ & Lab stock \\
\hline BW25113 & $\begin{array}{l}\text { F-, [?] (araD-araB)567, } \\
\text { [?]lacZ4787(::rrnB-3), -, rph-1, } \\
\text { [?](rhaD-rhaB)568, hsdR514 }\end{array}$ & (Datsenko and Wanner \\
\hline $\mathrm{BW}[?] l d h A$ & BW25113 ldhA null mutant & (Srirangan et al. 2014) \\
\hline DMH & BW[?]ldhA/pK-hemA & This study \\
\hline $\mathrm{DMH}[?] \operatorname{sdh} A$ & $s d h A$ null mutant of DMH $s d h A$ & This study This study \\
\hline $\mathrm{DMH}[?] \operatorname{sdh} A[?] i c l R$ & and $i c l R$ mutants of DMH & \\
\hline DMH-CT & $\begin{array}{l}\mathrm{DMH} / \mathrm{pK} \text {-hemA/pgRNA- } \\
\text { bacteria/pdcas9-bacteria }\end{array}$ & This study \\
\hline DMH-L1 & $\begin{array}{l}\text { DMH/pK-hemA/pgRNA- } \\
\text { L1/pdcas9-bacteria }\end{array}$ & This study \\
\hline DMH-L2 & $\begin{array}{l}\text { DMH/pK-hemA/pgRNA- } \\
\text { L2/pdcas9-bacteria }\end{array}$ & This study \\
\hline DMH-L3 & $\begin{array}{l}\text { DMH/pK-hemA/pgRNA- } \\
\text { L3/pdcas9-bacteria }\end{array}$ & This study \\
\hline DMH-L4 & $\begin{array}{l}\text { DMH/pK-hemA/pgRNA- } \\
\text { L4/pdcas9-bacteria }\end{array}$ & This study \\
\hline DMH-L4[?] sdhA & $s d h A$ null mutant of DMH-L4 & This study \\
\hline DMH-L4[?] iclR & $i c l R$ null mutant of DMH-L4 & This study \\
\hline
\end{tabular}




\begin{tabular}{|c|c|c|}
\hline Name & $\begin{array}{l}\text { Description, relevant } \\
\text { genotype or primer/oligo } \\
\text { sequence }\left(5,3^{\prime}\right)\end{array}$ & Source \\
\hline DMH-L4[?] sdhA[?] iclR & $\begin{array}{l}\text { sdh } A \text { and } i c l R \text { null mutants of } \\
\text { DMH-L4 }\end{array}$ & This study \\
\hline \multicolumn{3}{|l|}{ Plasmids } \\
\hline pCP20 pK184 & $\mathrm{Flp}+, \lambda \mathrm{cI} 857+, \lambda \mathrm{pR}$ & (Cherepanov and Wackernagel \\
\hline & Rep(pSC101 ori)ts, ApR, CmR & 1995) (Jobling and Holmes \\
\hline & p15A ori, KmR, Plac::lacZ' & \\
\hline pdcas9-bacteria & p15A ori, $\mathrm{P}_{\text {Tet }}$-dCas 9 & (Qi et al. 2013) \\
\hline pgRNA-bacteria & ColE1 origin, $\mathrm{P}_{\mathrm{J} 23119-\mathrm{gRNA}}$ & (Qi et al. 2013) \\
\hline pgRNA-L1 & $\begin{array}{l}\text { Derived from pgRNA-bacteria, } \\
\mathrm{P}_{\text {spel }}:: \text { hem } B \text {-gRNA-L1 }\end{array}$ & This study \\
\hline pgRNA-L2 & $\begin{array}{l}\text { Derived from pgRNA-bacteria, } \\
\mathrm{P}_{\mathrm{speI}}: \text { :hemB-gRNA-L2 }\end{array}$ & This study \\
\hline pgRNA-L3 & $\begin{array}{l}\text { Derived from pgRNA-bacteria, } \\
\mathrm{P}_{\text {speI }}: \text { hemB-gRNA-L3 }\end{array}$ & This study \\
\hline pgRNA-L4 & $\begin{array}{l}\text { Derived from pgRNA-bacteria, } \\
\mathrm{P}_{\text {speI }}: \text { hemB-gRNA-L4 }\end{array}$ & This study \\
\hline pK-hemA & $\begin{array}{l}\text { Derived from pTrc99a, } \\
\mathrm{P}_{t r c:}: \text { phaAB }\end{array}$ & This study \\
\hline \multicolumn{3}{|l|}{ Primers/oligo sequences } \\
\hline v-ldhA & \multicolumn{2}{|c|}{$\begin{array}{l}\text { GATAACGGAGATCGGGAATGAT(TAldwi et al. 2015) } \\
\text { GGTTTAAAAGCGTCGATGTCCAGTA }\end{array}$} \\
\hline v-sdhA & $\begin{array}{l}\text { CTCTGCGTTCACCAAAGTGT; } \\
\text { ACACACCTTCACGGCAGGAG }\end{array}$ & This study \\
\hline v-iclR & $\begin{array}{l}\text { GGTGGAATGAGATCTTGCGA; } \\
\text { CCGACACGCTCAACCCAGAT }\end{array}$ & This study \\
\hline c-frt & $\begin{array}{l}\text { AGATTGCAGCATTACACGTCTT } \\
\text { CCAGCTGCATTAATGAATCGG }\end{array}$ & $\begin{array}{l}\text { (Gisholiangan et al. 2014) } \\
\text {;CCATGGTCCATATGAATAT }\end{array}$ \\
\hline
\end{tabular}




\begin{tabular}{|c|c|c|}
\hline Name & $\begin{array}{l}\text { Description, relevant } \\
\text { genotype or primer/oligo } \\
\text { sequence }\left(5^{\prime}, 3^{\prime}\right)\end{array}$ & Source \\
\hline $\begin{array}{l}\text { c-ptrc cf-gRNA q-hemB q-rrsA } \\
\text { g-hemA g-pK-hemA g-pgRNA } \\
\text { hemA-gRNA-L1 } \\
\text { hemA-gRNA-L2 } \\
\text { hemA-gRNA-L3 } \\
\text { hemA-gRNA-L4 }\end{array}$ & \multicolumn{2}{|c|}{ 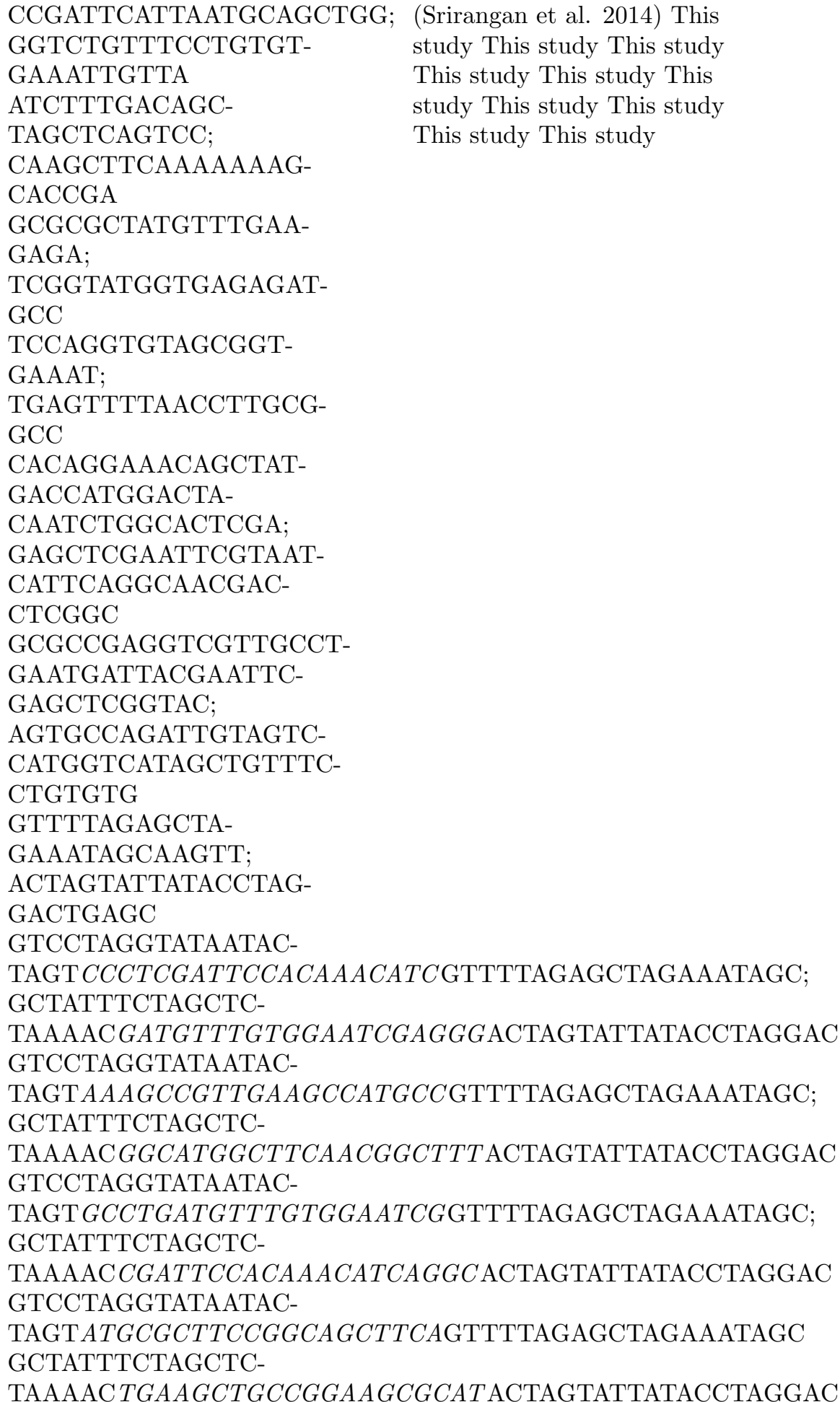 } \\
\hline
\end{tabular}



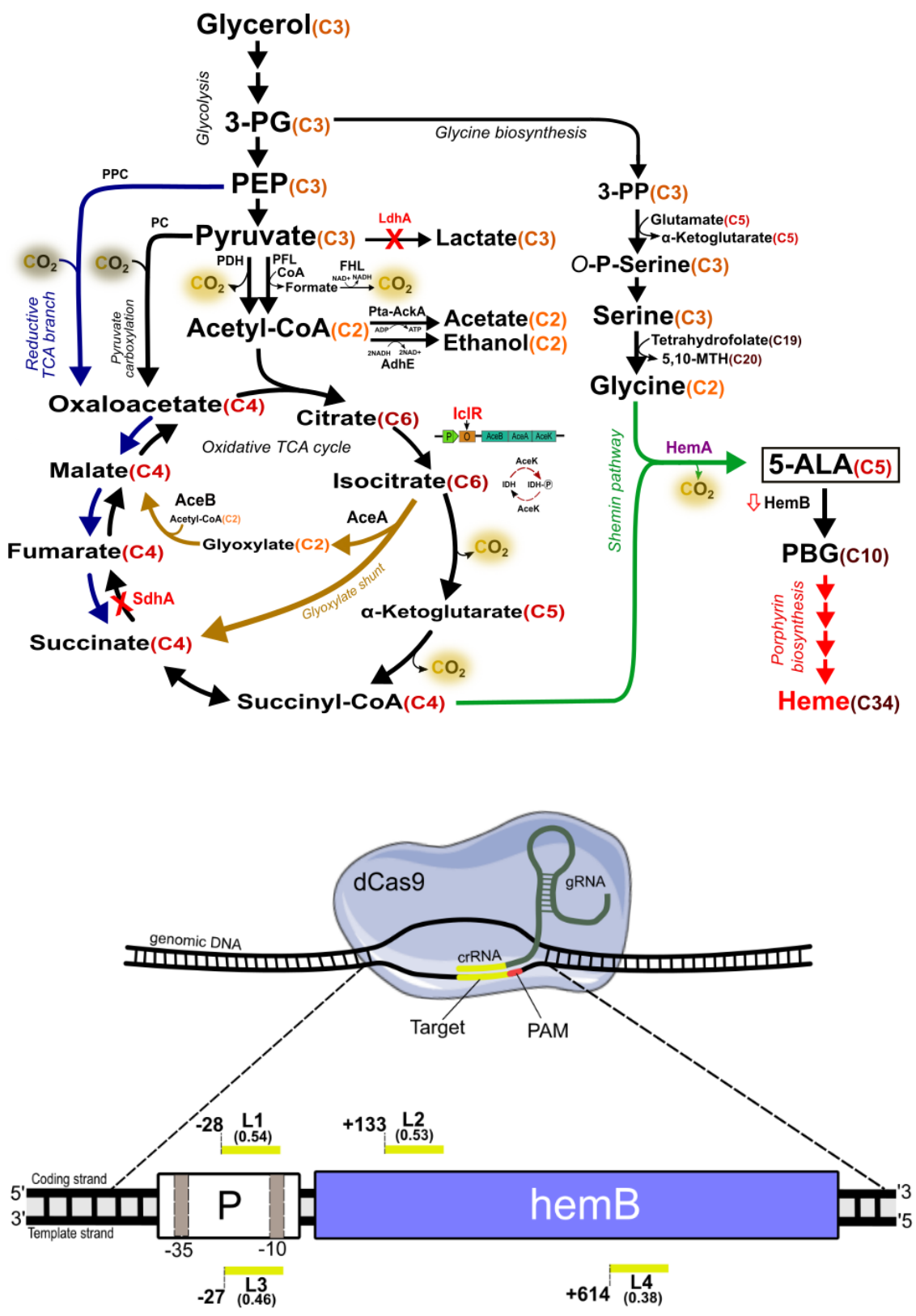


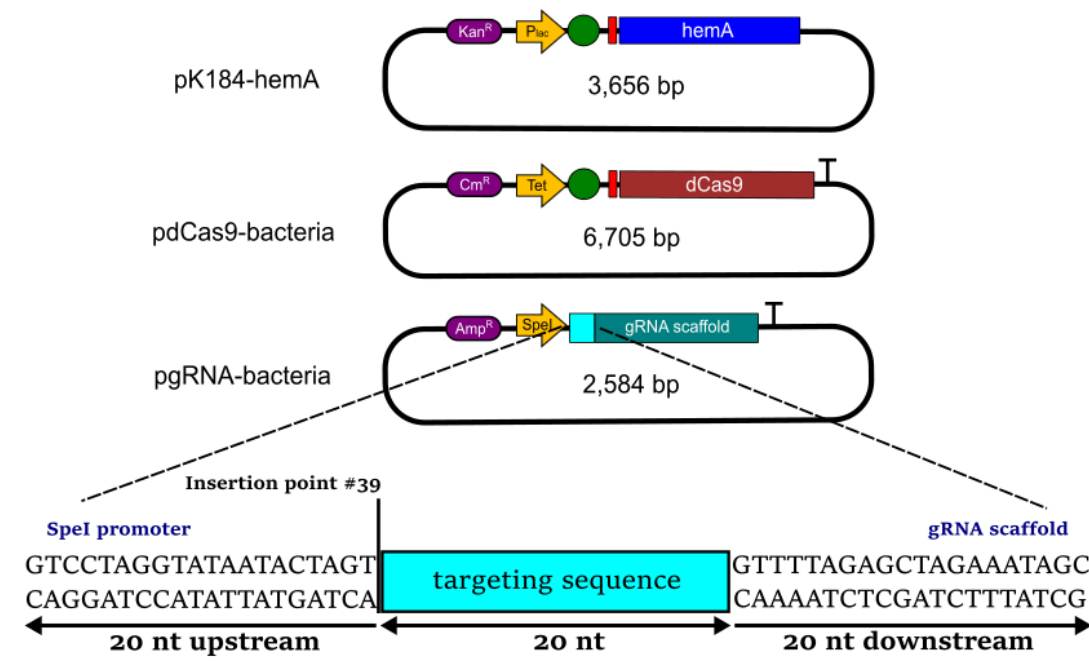

hemB-gRNA Gibson oligo design

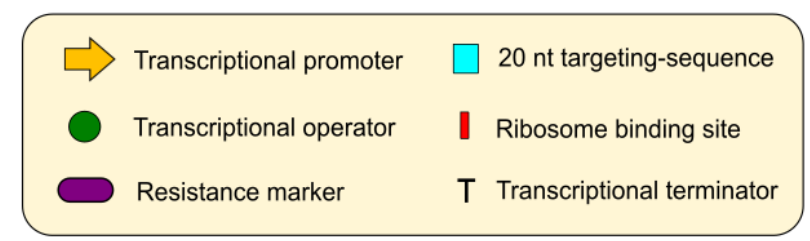

\begin{tabular}{ll}
\hline gRNA & 20 nt targeting-sequence \\
\hline hemB-gRNA-L1 & CCCTCGATTCCACAAACATC \\
hemB-gRNA-L2 & AAAGCCGTTGAAGCCATGCC \\
hemB-gRNA-L3 & GCCTGATGTTTGTGGAATCG \\
hemB-gRNA-L4 & ATGCGCTTCCGGCAGCTTCA \\
\hline
\end{tabular}

\section{Hosted file}

image4.emf available at https://authorea.com/users/302765/articles/432841-strain-engineeringfor-high-level-5-aminolevulinic-acid-production-in-escherichia-coli

Hosted file

image11.emf available at https://authorea.com/users/302765/articles/432841-strainengineering-for-high-level-5-aminolevulinic-acid-production-in-escherichia-coli

Hosted file

image14.emf available at https://authorea.com/users/302765/articles/432841-strainengineering-for-high-level-5-aminolevulinic-acid-production-in-escherichia-coli 